\title{
Domain and range operations in semigroups and rings
}

\author{
Tim Stokes
}

\begin{abstract}
A D-semigroup $S$ is a semigroup equipped with an operation $D$ satisfying laws asserting that for $a \in S, D(a)$ is the smallest $e$ in some set of idempotents $U \subseteq S$ for which $e a=a$. D-semigroups correspond to left-reduced $U$-semiabundant semigroups. The basic properties and many examples of D-semigroups are given. Also considered are D-rings, whose multiplicative semigroup is a D-semigroup. Rickart $*$-rings provide important examples, and the most general D-rings for which the elements of the form $D(a)$ constitute a lattice under the same meet and join operations as for Rickart *-rings are described.
\end{abstract}

\section{Introduction}

First some notation. Recall that a band is a semigroup in which every element is idempotent, so that $x^{2}=x$ is a law. We use the term "semilattice" for both commutative bands and posets on which the least upper bound of any two elements exists; context will indicate which meaning is intended. The set of all idempotents on a semigroup $S$ is denoted by $E(S)$, which is partially ordered if we define $e \leq f$ if and only if $e=e f=f e$, and we call this the standard order on $E(S)$ (and indeed on any subset of $E(S)$ ). The set of central idempotents in the semigroup $S$ is $C(S)=\{e \in E(S) \mid e a=a e$ for all $a \in S\}$, a subsemigroup of $S$ which is a semilattice.

An involution on a semigroup or ring $S$ is a unary operation * for which, for all $a, b \in S$, $\left(a^{*}\right)^{*}=a,(a b)^{*}=b^{*} a^{*}$, and in the ring case, $(a+b)^{*}=a^{*}+b^{*}$. Denote by $E^{*}(S)$ the set of all symmetric idempotents: those $e \in E(S)$ for which $e^{*}=e$.

If $X$ is a set, $\mathcal{R}(X)$ denotes the semigroup of binary relations on $X, \mathcal{P}(X)$ the semigroup of partial functions $X \rightarrow X$, and $\mathcal{T}(X)$ the semigroup of transformations on $X$. For $\rho \in \mathcal{R}(X)$, define $\operatorname{dom}(\rho)=\{x \in X \mid(x, y) \in \rho$ for some $y \in \rho\}$ (the usual definition of domain if $\rho \in \mathcal{P}(X))$, and define $\operatorname{ran}(\rho)$ dually.

In a ring $A$, the circle composition operation $\circ$ is given by $a \circ b=a+b-a b$ for all $a, b \in A$, and is associative.

We say the semigroup $S$ is a $D$-semigroup if there exists some $U \subseteq E(S)$ such that, for all $a \in S$, there is a smallest $e \in U$ (with respect to the standard order) with the property that $e a=a$, and then we call this element $D(a)$. It is clear that $D(S)=\{D(a) \mid a \in S\}=U$.

In a D-semigroup, the mapping $a \mapsto D(a)$ determines a unary operation $D$ on $S$, and the following is obvious.

Proposition 1.1 Let $S$ be a D-semigroup with respect to $U \subseteq E(S)$. Then $S$ satisfies the following laws: for all $a, b \in S$, 


$$
\begin{aligned}
& \text { 1. } D(a) a=a \\
& \text { 2. } D^{2}(a)=D(a) \\
& \text { 3. } D(a b) D(a)=D(a) D(a b)=D(a b) \text {. }
\end{aligned}
$$

In fact these laws characterise D-semigroups.

Proposition 1.2 Suppose $S$ is a unary semigroup satisfying the three laws listed in the previous result. Then $S$ is a D-semigroup with respect to $D(S)=\{D(a) \mid a \in S\} \subseteq E(S)$.

Proof. From the first law, we obtain $D(D(a)) D(a)=D(a)$, so from the second, $D(a)^{2}=$ $D(a)$. Hence $D(S)=\{D(s) \mid s \in S\} \subseteq E(S)$. If $e a=a$ for some $e \in D(S)$, then $e D(a)=e D(e a)=D(e) D(e a)=D(e a)=D(a)$, and similarly $D(a) e=D(a)$, so $D(a) \leq e$. So by the first law, $D(a)$ is the smallest element $e$ of $D(S)$ for which $e a=a$.

With $D$ viewed as an operation in this way, the class of D-semigroups is a finitely based variety of unary semigroups. An important subvariety is defined by the additional law $D(a) D(b)=D(b) D(a)$, which is easily seen to be equivalent to assuming that $D(S)$ is a semilattice. These objects are called type SL $\gamma$-semigroups in [2] and left C-semigroups in [19].

D-semigroups arise as one-sided versions of the $U$-semiabundant semigroups discussed in [24]. These are first introduced in [23], where a connection with Rees matrix semigroups is made. To define $U$-semiabundant semigroups, we must define the following generalised Green's relation on the semigroup $S$, in terms of $U \subseteq E(S)$ :

$$
(x, y) \in \overline{\mathcal{R}} \text { if for all } e \in U, e x=x \Leftrightarrow e y=y .
$$

Now $\overline{\mathcal{R}}$ is an equivalence relation, and $S$ is said to be left $U$-semiabundant if each $\overline{\mathcal{R}}$-class contains at least one element of $U$.

If $S$ is a semigroup, we say $U \subseteq E(S)$ is left-reduced if for all $e, f \in U, e=f e \Rightarrow e=e f$. Suppose $S$ is a left $U$-semiabundant semigroup and $U$ is left-reduced. Then each $\overline{\mathcal{R}}$-class contains a unique element of $U$, as follows from the arguments given in [24], easily adapted to the one-sided case. If then $e$ is the unique element of $U$ in the $\overline{\mathcal{R}}$-class containing $a$, then, as in Lemma 1.2 in [24], $e a=a$, and for all $f \in U, f a=a \Leftrightarrow f e=e$; but $f e=e$ implies $e f=e$, so $e \leq f$. So $e \in \overline{\mathcal{R}}_{a}$ is the smallest $f \in U$ for which $f a=a$, and so $S$ is a D-semigroup with $D(S)=U$. Conversely, it is just as easy to see that a D-semigroup is a left $D(S)$-semiabundant semigroup in which $D(S)$ is left-reduced, and $D(a)$ is the unique element of $D(S)$ in the $\overline{\mathcal{R}}$-class containing $a$. Summarising, we have the following.

Proposition 1.3 Let $S$ be a semigroup, with $U \subseteq E(S)$. Then $S$ is a D-semigroup in which $D(S)=U$ if and only if $S$ is a left $U$-semiabundant semigroup in which $U$ is left-reduced.

The idea is easily dualised. An $R$-semigroup is a semigroup $S$ for which there exists some $U \subseteq E(S)$ such that, for all $a \in S$, there is a smallest $e \in U$ (with respect to the standard order) with the property that $a e=a$. These are characterised by the obvious right-sided variants of the laws given in Proposition 1.1. There is also a characterisation along the lines 
of Proposition 1.3, in terms of the notion of right $U$-semiabundant semigroups in which $U$ is right-reduced ( $e f=e$ implies $f e=e$ for all $e, f \in U$ ), defined using a generalised Green's relation $\overline{\mathcal{L}}$ which is a left-sided version of $\overline{\mathcal{R}}$ given above.

In a D-semigroup $S, D(S)$ is left-reduced though not necessarily right-reduced. If it is both we say $D(S)$ is reduced, and we also simply say that the D-semigroup $S$ is reduced. Similarly for an R-semigroup $S$ in which $R(S)$ is both left- and right-reduced.

A DR-semigroup is a semigroup which is both a D-semigroup and an R-semigroup relative to the same set of idempotents $U$. These may be characterised equationally using the union of the D-semigroup and R-semigroup laws, along with the following pair:

$$
D(R(a))=R(a), R(D(a))=D(a) \text { for all } a \in S .
$$

DR-semigroups are exactly reduced $U$-semiabundant semigroups in the sense of [24] in which $D, R$ are made into unary operations.

In [24], the $U$-semiabundant semigroup $S$ is said to be $U$-semiadequate if $e f=f e$ for all $e, f \in U$. Obviously this property implies that $S$ is reduced. Again, there are obvious onesided variants, giving rise to the notions of left and right $U$-semiadequate semigroups. In the language of D-semigroups, this property is obviously expressible as $D(a) D(b)=D(b) D(a)$, which implies that $D(S)$ is a semilattice, and we are back to the type SL $\gamma$-semigroups of [2] and the left C-semigroups of [19]. Here, we shall call a D-semigroup D-semiadequate if it satisfies the law $D(a) D(b)=D(b) D(a)$.

Many examples and subclasses of D-semiadequate D-semigroups are considered in [19]. Some of the most important D-semiadequate DR-semigroups are those in which $D(a)$ and $R(a)$ model notions of domain and range for certain types of generalised functions (hence the use of " $D$ " and " $R$ " throughout this article). Thus if $D(f)$ is defined to be the subset of the diagonal relation (restriction of the identity map) on $X$ to the domain of the partial transformation $f \in \mathcal{P}(X)$ on $X$, then $\mathcal{P}(X)$ is a D-semiadequate DR-semigroup in which $D(\mathcal{P}(X))$ consists of all restrictions of the identity function; dually for range. These operations also make sense for the semigroup of binary relations on a set, on which the operation $D$, as well as $D$ and $R$ together, have been studied in a computer science setting in [27].

The D-semiadequate semigroups embeddable in $\mathcal{P}(X)$ for some $X$ can be specified by one additional equation (namely, $a D(b)=D(a b) a$ ), thereby giving the variety of left restriction semigroups, considered by a great many authors and under many different names; see [34] and [36] where the axiomatization was first shown to be complete, [19] and [26] where this was re-discovered, as well as [12] and [13], where they arise as weakly left Eample semigroups. The axioms for those D-semiadequate DR-semigroups embeddable in $\mathcal{P}(X)$ equipped with both domain and range operations were provided by [32], following a correction to a result in [34].

Restriction semigroups are themselves generalisations of inverse semigroups in which one defines $D(a)=a a^{\prime}$ (and $R(a)=a^{\prime} a$ ), where $a^{\prime}$ is the inverse of $a$; the Vagner-Preston Theorem ([29], [37], [38]), shows that $D, R$ can in this case be interpreted as nothing but domain and range operations for semigroups of injective partial functions. A categorical version is considered in [5] in the setting of "restriction categories". In the one-sided case, a type of "one-sided inductive category" is developed in [13], and restriction semigroups are characterised in terms of them. A version in which the domain elements constitute a 
Boolean algebra, with connections to the algebra of computer programs, is considered in [21].

In the setting of associative rings, a (left-sided versions of a) closure ring as considered in [11] is a ring $A$ whose multiplicative semigroup is D-semiadequate, so that $D(A)$ is a semilattice under multiplication, but also such that $D(A)$ is closed under the circle composition operation $\circ$ (given by $a \circ b=a+b-a b$ ). It follows that $D(A)$ is a distributive lattice in which meet is multiplication and join is $\circ$.

But there are also many natural examples of D-semigroups and DR-semigroups which are not (left) $U$-semiadequate. One of the most natural arises from the multiplicative semigroup of a certain type of associative ring. A (left) Rickart *-ring is a ring with involution in which the left annihilator of every element is generated as a left ideal by a symmetric idempotent element (often called a projection in this setting). The main motivating example is the ring of all bounded linear operators on a Hilbert space, in which the involution of an element is its adjoint. This notion was first introduced by Maeda in [25].

A basic fact about a Rickart *-ring $A$ is that for every $a \in A$, there are projections $L P(a), R P(a)$ such that $L P(a) \cdot a=a$ and $a \cdot R P(a)=a$, and moreover these are respectively the smallest projections $e, f$ for which $e a=a=a f$, under the standard order on the set of projections. (See for example the first two pages of [3].) This means that the multiplicative semigroup of $A$ is a DR-semigroup, and one which is not generally D-semiadequate. In the Rickart *-ring $L(H)$ of all bounded linear operators on the Hilbert space $H$, for any $f \in$ $L(H), D(f)=L P(f)$ is the (orthogonal) projection of $f$ onto the orthogonal complement of the null space of $f$ (a reasonable analog of domain), and $R(f)=R P(f)$ is the orthogonal projection onto the image of $f$. We return to this example later.

Any left zero semigroup is a D-semigroup, in which one defines $D(a)=a$ for all $a \in S$. This is easily seen to satisfy the axioms, and $D(S)=S$. In fact $D(S)$ is flat under the standard partial order, and indeed $a \in S$ is the only $e \in D(S)=S$ for which $e a=a$, hence the smallest. Clearly this example is not D-semiadequate, nor is it a DR-semigroup.

Generalising a definition from [19], we say the D-semigroup $S$ is full if $D(S)=E(S)$. It is of interest to determine those semigroups that are full D-semigroups. A necessary condition is that $E(S)$ be left-reduced; in the two-sided case, it must be reduced.

Every left zero semigroup is full as a D-semigroup. Inverse semigroups are full DRsemigroups. Indeed if in a regular semigroup $S$ it happens that $E(S)$ is reduced, then $S$ is inverse; see [16]. So in particular, a regular DR-semigroup is full if and only if it is inverse. On the other hand, every left zero semigroup is a regular full D-semigroup which is not reduced.

It also follows that none of $\mathcal{T}(X), \mathcal{P}(X), \mathcal{R}(X)$ is full if $|X|>1$. For suppose $\{x, y\} \subseteq X$ with $x \neq y$. Then $e=\{(x, x),(y, x)\}$ and $f=\{(x, y),(y, y)\}$ are evidently idempotent in $\mathcal{P}(X) \subseteq \mathcal{R}(X)$, and satisfy ef $=f, f e=e$.

The remainder of this paper is structured as follows. First we consider some of the most important special kinds of D-semigroups, defined by the congruence conditions which feature in Lawson's work on the D-semiadequate case in [24]. These are shown to arise in many different ways. Attention then turns to D-semigroups having an involution operation under 
which $D(S)$ is closed. The notion of a $*$-full involuted D-semigroup (in which $D(S)=E^{*}(S)$ ) is introduced. It is shown that although it is not full, $\mathcal{R}(X)$ is $*$-full (where $*$ is relational converse). It is also shown that all DR-semigroups arise from such involuted D-semigroups. Versions of the semigroup results for the case of rings are then considered: many of the properties coincide or have simpler forms in the ring setting. In the D-semiadequate case, further simplifications occur. One of the important properties of a Rickart *-ring $A$ is that $D(A)$ constitutes a lattice (indeed an orthomodular one) under operations expressible in the language of D-rings, and in the final section we determine the most general D-ring setting in which $D(A)$ is a lattice under these operations. Some subdirect product representations are then given in the $\mathrm{D}$-semiadequate case.

\section{The congruence conditions and related properties}

\subsection{The left and right congruence conditions}

We say the D-semigroup $S$ satisfies the left congruence condition if $D(a b)=D(a D(b))$ for all $a, b$. There is an obvious right congruence condition for R-semigroups. A DR-semigroup satisfying both is said to satisfy the congruence condition. As in [24], the left congruence condition is equivalent to $\overline{\mathcal{R}}$ being a left congruence, and dually for the right congruence condition with respect to $\overline{\mathcal{L}}$.

Because in any D-semigroup, $D(a D(b)) a b=D(a D(b)) a D(b) b=a D(b) b=a b$, we always have $D(a b) \leq D(a D(b))$. So the left congruence condition is equivalent to the apparently weaker law $D(a D(b)) \leq D(a b)$.

The congruence conditions feature heavily in the D-semiadequate cases considered in the literature. The D-semiadequate DR-semigroup $\mathcal{R}(X)$, in which $D(\mathcal{R}(X))$ is all subsets of the diagonal relation, satisfies both congruence conditions, hence so does any DR-subsemigroup of it (hence all inverse semigroups with $D(a)=a a^{\prime}$ and $R(a)=a^{\prime} a$ for all $a$, and more generally all two-sided restriction semigroups). The domain semirings considered in [27] (where algebras of binary relations are modelled) satisfy the left congruence condition. Further examples are given in [24], which concentrates mainly on D-semiadequate DRsemigroups satisfying the congruence condition. Shortly we encounter non-D-semiadequate examples that arise from the multiplicative semigroups of rings.

On the other hand, examples of D-semiadequate D-semigroups not satisfying the left congruence condition are given in [19]. A non-D-semiadequate example is the rectangular band on the set $X \times X$ on which we define $(a, b)(c, d)=(a, d)$ for all $a, b, c, d \in X$ : defining $D((a, b))=(a, a)$ and $R((a, b))=(b, b)$ gives a DR-semigroup not satisfying either congruence condition.

DR-semigroups satisfying the congruence condition can be viewed as (small) categories, as in [24]. Indeed it follows easily from Theorem 1.7 in [24] that there is a functor from the category of DR-semigroups satisfying the congruence condition (having as arrows all DR-semigroup homomorphisms) to the category of small categories and functors. In [24], D-semiadequate DR-semigroups satisfying the congruence condition are called Ehresmann semigroups, and are characterised in terms of certain types of ordered categories. 


\subsection{D-abundant D-semigroups}

Historically, the notion of a semiabundant semigroup, which is a semigroup $S$ that is an $E(S)$-semiabundant semigroup, preceded that of a general $U$-semiabundant semigroup. Semiabundant semigroups were introduced by El-Qallali in [7], and are themselves generalisations of both regular semigroups and abundant semigroups, the latter first studied by Fountain [9]. The original abundant semigroup notion is two-sided, but there are obvious one-sided definitions, which we turn to next.

We say that a semigroup $S$ is left-abundant if every $\mathcal{R}^{*}$-class of $S$ contains at least one idempotent. Here $\mathcal{R}^{*}$ is a generalised Green's relation (equivalently) defined as follows: $(a, b) \in \mathcal{R}^{*}$ providing

$$
\text { for all } x, y \in S^{1}, x a=y a \text { if and only if } x b=y b \text {. }
$$

(Here $S^{1}$ is the semigroup $S$ with an identity adjoined.) Evidently $\mathcal{R}^{*} \subseteq \overline{\mathcal{R}}$ (where $\overline{\mathcal{R}}$ is defined relative to some $U \subseteq E(S))$. If $e \in E(S)$, then $(a, e) \in \mathcal{R}^{*}$ if and only if $e a=a$ and for all $x, y \in S^{1}, x a=y a$ implies $x e=y e$. The notion of right-abundant is defined using the equivalence relation $\mathcal{L}^{*}$, which is defined dually to $\mathcal{R}^{*}$; and "abundant" = "left abundant + right abundant".

As Theorem 1.5 of [24] shows (itself based on results in [10]) and [16], abundant semigroups satisfy $\overline{\mathcal{R}}=\mathcal{R}^{*}$ and $\overline{\mathcal{L}}=\mathcal{L}^{*}$ (where $U=E(S)$ ), and so are semiabundant, satisfying the congruence condition (meaning that $\overline{\mathcal{R}}$ is a left congruence and $\overline{\mathcal{L}}$ a right congruence). But they need not be D-semigroups.

In [1] an abundant semigroup is called amiable if there is a unique idempotent in every $\mathcal{L}^{*}$-class and every $\mathcal{R}^{*}$-class. In Table 1 on page 4441 of [1], a 4-element amiable semigroup $S$ is given. By the previous comments this semigroup must be semiabundant, and because $E(S)$ is reduced (as is easily checked), it is a full DR-semigroup satisfying the congruence conditions. Note that it is not D-semiadequate.

A generalisation beckons. We say the semigroup $S$ with $U \subseteq E(S)$ is left $U$-abundant if every $\mathcal{R}^{*}$-class of $S$ contains at least one idempotent in $U$; define the terms right $U$-abundant and $U$-abundant in the obvious ways. Because $\mathcal{R}^{*} \subseteq \overline{\mathcal{R}}$, it is immediate that if $S$ is left $U$-abundant, then it is left $U$-semiabundant. The next result then follows immediately.

Proposition 2.1 Let $S$ be a semigroup with $U \subseteq E(S)$ left-reduced. If $S$ is left $U$-abundant, then $S$ is a D-semigroup with $D(S)=U$.

We call a D-semigroup as in this last result D-abundant. There are obvious definitions for R-abundant and DR-abundant.

Proposition 2.2 Let $S$ be a D-semigroup. The following are equivalent.

1. $S$ is D-abundant.

2. For all $x, y \in S^{1}, x a=y a$ implies $x D(a)=y D(a)$.

In this case, $S$ satisfies the left congruence condition. 
Proof. $\quad(1) \Rightarrow(2)$ is immediate. Conversely, if (2) holds, then since $D(a) a=a$, it is obvious that $(a, D(a)) \in \mathcal{R}^{*}$, and so $S$ is D-abundant.

Under these conditions, for all $a, b \in S$, because $D(a b) a b=a b$, it follows that $D(a b) a D(b)=$ $a D(b)$, so $D(a D(b)) \leq D(a b)$, establishing the left congruence condition.

The law in (2) of Proposition 2.2 is not necessary for the left congruence condition. For example, if $S=\mathcal{R}(X)$, the D-semiadequate D-semigroup of binary relations on $X$, with $D(\rho)$ equal to the restriction of the diagonal to $\operatorname{dom}(\rho),(2)$ fails (as easy examples show). In fact (2) holds on the sub-D-semigroup of injective partial functions in $\mathcal{R}(X)$.

The following proves useful.

Lemma 2.3 Let $S$ be a D-abundant D-semigroup. Then for $e \in E(S)$, eD $(e)=D(e)$.

Proof. For $e \in E(S), e=e^{2}=D(e) e$, so $e D(e)=D(e) D(e)=D(e)$ by Proposition 2.2.

Corollary 2.4 Let $S$ be a D-abundant D-semigroup. Then every idempotent commuting with all of $D(S)$ is itself in $D(S)$, so in particular $C(S) \subseteq D(S)$.

Proof. If $e \in E(S)$ commutes with all of $D(S)$, then $e=D(e) e=e D(e)=D(e)$ by Lemma 2.3, so $e \in D(S)$.

\subsection{Strong D-semigroups}

A D-semigroup $S$ is strong if for all $a \in S$ there exists $b \in S$ for which $a b=D(a)$. Evidently this is true for inverse semigroups (let $b=a^{\prime}$ for example) and implies regularity. An Rsemigroup is strong if for all $a \in S$ there is $b \in S$ such that $b a=R(a)$, and a DR-semigroup is strong if it is strong as both a D-semigroup and an R-semigroup. These properties were explored in the D-semiadequate case in [19], and the following simplification of axioms adapts easily from that case.

Proposition 2.5 Suppose the semigroup $S$ equipped with the unary operation D satisfies the following laws:

$$
\begin{aligned}
& \text { 1. } D(a) a=a \\
& \text { 2. } D(a b)=D(a b) D(a) \text {; } \\
& \text { 3. for all } a \in S \text { there exists } b \in S \text { such that } a b=D(a) \text {. }
\end{aligned}
$$

Then $S$ is a strong D-semigroup.

Proof. Let $a, b \in S$. Then there exist $a^{\prime}, b^{\prime}, c \in S$ such that $a a^{\prime}=D(a), b b^{\prime}=D(b)$ and $a b c=D(a b)$. Then $D(a) D(a b)=D(a) a b c=a b c=D(a b)$. Also,

$$
D(b)=D(D(b)) D(b)=D\left(b b^{\prime}\right) D(b)=D\left(b b^{\prime}\right)=D(D(b)) .
$$

So all the D-semigroup laws are satisfied.

Next is a fact not observed in [19], even for the D-semiadequate case considered there. 
Proposition 2.6 Every strong D-semigroup satisfies the left congruence condition.

Proof. Let $b^{\prime} \in S$ be such that $b b^{\prime}=D(b)$. Then $D(a D(b))=D\left(a b b^{\prime}\right) \leq D(a b)$.

So every strong D-semigroup is regular and satisfies the left congruence condition. The converse fails: the full transformation semigroup $\mathcal{T}(X)$ on the set $X$ is a regular restriction semigroup (indeed a monoid) in which $D(a)=1$ for all $a$, which clearly satisfies the left congruence condition, but this D-semigroup is not strong since most of its elements do not have right inverses.

\subsection{Connection with first projection quasi-orders}

The relation $\triangleleft$ on $\mathcal{R}(X)$, defined by $\rho \triangleleft \theta$ if and only if $\operatorname{dom}(\rho) \subseteq \operatorname{dom}(\theta)$, is a quasiorder (a reflexive transitive relation) and, as shown by Schein, is otherwise axiomatized on semigroups of binary relations by the two laws

- $\rho \theta \triangleleft \rho$ for all $\rho, \theta \in \mathcal{R}(X)$ and

- if $\rho_{1} \triangleleft \rho_{2}$ then $\theta \rho_{1} \triangleleft \theta \rho_{2}$ for all $\rho_{1}, \rho_{2}, \theta \in \mathcal{R}(X)$.

The same laws axiomatize semigroups of partial functions equipped with this quasi-order. (See [30] or [33].) A quasi-order satisfying these two laws on an abstract semigroup is called a first projection quasi-order.

If we define the binary relation $\triangleleft$ on the D-semigroup $S$ by $a \triangleleft b$ if and only if $D(a) \leq$ $D(b)$, it is clear that $\triangleleft$ is a quasi-order on $S$, satisfying $a b \triangleleft a$ for all $a, b \in S$ (indeed this condition is equivalent to the law $D(a b) \leq D(a)$ for D-semigroups).

Proposition 2.7 Let $S$ be a D-semigroup with $\triangleleft$ defined as above on $S$. Then $\triangleleft$ is a first projection quasi-order on $S$ if and only if $S$ satisfies the left congruence condition.

Proof. Let $S$ be a D-semigroup. Suppose it satisfies the left congruence condition. Defining $a \triangleleft b$ as indicated, if $a_{1} \triangleleft a_{2}$ then $D\left(a_{1}\right) \leq D\left(a_{2}\right)$, and so

$$
D\left(b D\left(a_{2}\right)\right) b D\left(a_{1}\right)=D\left(b D\left(a_{2}\right)\right) b D\left(a_{2}\right) D\left(a_{1}\right)=b D\left(a_{2}\right) D\left(a_{1}\right)=b D\left(a_{1}\right),
$$

and so $D\left(b a_{1}\right)=D\left(b D\left(a_{1}\right)\right) \leq D\left(b D\left(a_{2}\right)\right)=D\left(b a_{2}\right)$, so $b a_{1} \triangleleft b a_{2}$. So $\triangleleft$ is a first projection quasi-order.

Conversely, suppose $\triangleleft$ is a first projection quasi-order on $S$. Then for $a, b \in S$, $D(D(b))=D(b)$, so in particular, $D(b) \triangleleft b$, and so $a D(b) \triangleleft a b$, so that $D(a D(b)) \leq D(a b)$.

\subsection{Congruences}

A congruence on a D-semigroup is assumed to be compatible with $D$ as well as with the semigroup operation; likewise for R-semigroups and DR-semigroups. We next generalise Proposition 4.2 of [19], itself a generalisation of a known result for inverse semigroups relating congruences and traces. 
For $S$ a D-semigroup, define the trace of the congruence $\theta$ on $S$ to be its restriction to $D(S)$; since $D(S)$ is a subalgebra, the trace of $\theta$ is a congruence on it. In general, if $\rho$ is a congruence on $D(S)$, we say $\rho$ is normal if it satisfies the following condition: for all $e, f \in D(S)$ and $x \in S$,

$$
\text { e } \rho f \Rightarrow D(x e) \rho D(x f) \text { and } D(e x) \rho D(f x) .
$$

It is easy to see that the trace of any congruence on $S$ is a normal congruence on $D(S)$.

This use of the term "normal congruence" is consistent with its usage for left Csemigroups as in [19], at least if the left congruence condition is assumed. In [19], the definition given was: for all $a, b, x \in S$,

$$
D(a) \rho D(b) \Rightarrow D(x a) \rho D(x b) .
$$

However, this condition is equivalent to the condition $e \rho f \Rightarrow D(x e) \rho D(x f)$ if the left congruence condition is assumed, and in the D-semiadequate case considered in [19], the other half is immediate if the left congruence condition is assumed because $D(e x)=e D(x)$, and so $e \rho f \Rightarrow D(e x) \rho D(f x)$.

Let $S$ be a D-semigroup. For $\rho$ a normal congruence on $D(S)$, define

$$
\rho_{m}=\{(a, b) \in S \times S \mid D(a) \rho D(b) \text { and for all } e \in D(S), D(a e) \rho D(b e)\} .
$$

Theorem 2.8 Suppose $S$ satisfies the left congruence condition. The relation $\rho_{m}$ as just defined is the largest congruence on $S$ having trace $\rho$.

Proof. The proof follows similar lines to that of Proposition 4.2 in [19]. Thus, $\rho_{m}$ is evidently an equivalence relation on $S$, moreover one respecting $D$ : for if $a \rho_{m} b$ then $D(a) \rho D(b)$, so $D(D(a)) \rho D(D(b))$, and so because $\rho$ is normal, for all $e \in D(S)$ we have $D(D(a) e) \rho D(D(b) e)$, and so $D(a) \rho_{m} D(b)$.

Suppose $(a, b) \in \rho_{m}$ with $x \in S$. Then $D(a) \rho D(b)$ and $D(a e) \rho D(b e)$ for all $e \in D(S)$, so by normality of $\rho$ and the left congruence condition, $D(x a)=D(x D(a)) \rho D(x D(b))=$ $D(x b)$, and similarly, for all $e \in D(S), D(x a e)=D(x D(a e)) \rho D(x D(b e))=D(x b e)$, so by definition, $x a \rho_{m} x b$. Also, for all $e \in D(S), D($ axe $)=D(a D(x e)) \rho D(b D(x e))=D(b x e)$, and also $D(a x)=D(a D(x)) \rho D(b D(x))=D(b x)$. So $a x \rho_{m} b x$. So $\rho_{m}$ is a D-semigroup congruence.

We show $\rho_{m}$ has trace $\rho$. If $e \rho f$ then $D(e)=e \rho f=D(f)$, and for all $g \in D(S)$, $D(e g) \rho D(f g)$ by normality of $\rho$, so $e \rho_{m} f$. Conversely, if $e, f \in D(S)$ are such that e $\rho_{m} f$, then $e=D(e) \rho D(f)=f$.

Finally, we show $\rho_{m}$ is the largest congruence with trace $\rho$. Suppose $\theta$ is another such, with $a \theta b$. Then $D(a) \theta D(b)$, so $D(a) \rho D(b)$. Also, for all $e \in D(R)$, ae $\theta$ be, so $D(a e) \theta D(b e)$, and so $D(a e) \rho D(b e)$. So $a \rho_{m} b$ by definition. Hence $\theta \subseteq \rho_{m}$.

In the special case that $\rho=\triangle$, the diagonal relation on $D(S), \rho_{m}$ is the largest congruence on $S$ separating elements of $D(S)$. 


\subsection{Normality}

The left congruence condition can be usefully weakened somewhat. We say the D-semigroup $S$ is (left) normal if for all $a \in S$ and $e \in D(S), D(e a)=D(e D(a)$ ). (Note that this usage of the word "normal" is unrelated to the one applying to congruences used above.) Evidently the left congruence condition implies normality (we omit "left" if the context is clear). In [20], this property was defined as $e D(a)=D(e a)$, which is easily seen to be equivalent to the current definition in the D-semiadequate case considered there, but is not in general (since it implies that $D(S)$ is closed under multiplication). Any direct product of monoids with zero is a normal D-semiadequate D-semigroup if $D\left(\left(a_{i}\right)\right)=\left(e_{i}\right)$, where $e_{i}=0$ if $a_{i}=0$ and $e_{i}=1$ otherwise; generally such examples do not satisfy the left congruence condition. The normality property will feature in our work on rings.

\section{Involuted D-semigroups}

Involutions on semigroups are common. Relational converse (given by $(x, y) \in \rho^{*}$ if and only if $(y, x) \in \rho)$ is an involution on $\mathcal{R}(X)$. The inverse operation on an inverse semigroup is an involution. Transposition on the semigroup under multiplication of $n \times n$ matrices over a ring is an involution.

A semigroup with involution is a semigroup $S$ equipped with an involution, viewed as a unary semigroup. The following is routine to verify.

Proposition 3.1 Let $S$ be a D-semigroup which is also a semigroup with involution. Then defining $R(a)=D\left(a^{*}\right)^{*}$ makes $S$ an $R$-semigroup. This definition makes $S$ a DR-semigroup if and only if $e^{*} \in D(S)$ for all $e \in D(S)$ (equivalently, $D\left(D(a)^{*}\right)=D(a)^{*}$ for all $a \in S$ ), and then $R(a)=D\left(a^{*}\right)$ for all $a \in S$.

Because of this last result, if $S$ is a D-semigroup which is also a semigroup with involution *, we call it involuted if $D\left(D(a)^{*}\right)=D(a)^{*}$ for all $a \in S$. Every involuted D-semigroup is to be thought of as a DR-semigroup as in Proposition 3.1. If an involuted D-semigroup satisfies the left congruence condition then it satisfies both congruence conditions when viewed as a DR-semigroup; similarly for normality.

If an involuted D-semigroup satisfies the generally stronger condition that $D(a)^{*}=D(a)$ for all $a \in S$, we call it a symmetric involuted D-semigroup.

We say an involuted D-semigroup $S$ is $*$-full if $D(S)=E^{*}(S)$; of course such $S$ will be symmetric. Inverse semigroups are $*$-full involuted D-semigroups (and indeed are full since $E^{*}(S)=E(S)$ ). The multiplicative D-semigroup of a Rickart $*$-ring is $*$-full, as noted earlier; indeed this is a special case of the following useful fact.

Lemma 3.2 Every symmetric involuted D-abundant D-semigroup is $*$-full.

Proof. Let $S$ be a symmetric involuted D-abundant D-semigroup. Of course $D(S) \subseteq$ $E^{*}(S)$ by symmetry. Conversely, let $e \in E^{*}(S)$. Then $D(e)=e D(e)$ by Lemma 2.3 , so $D(e)^{*}=D(e)^{*} e^{*}$, and so $D(e)=D(e) e=e$, so $e \in D(S)$.

The D-semiadequate D-semigroup $\mathcal{R}(X)$ on which $D(\rho)$ is the restriction of the diagonal relation to $\operatorname{dom}(\rho)$ is symmetric involuted, hence is not D-abundant by the previous 
lemma, since $D(\mathcal{R}(X)) \neq E^{*}(\mathcal{R}(X))$. Those D-semigroups (and indeed DR-semigroups) embeddable in $\mathcal{R}(X)$ admit no finite axiomatization; see Theorem 10.1 of [22]. However, those ordered involuted semigroups embeddable in $\left(\mathcal{R}(X), \cdot, D, R,{ }^{*}, \subseteq\right)$, where ${ }^{*}$ is relational converse and $\subseteq$ denotes set inclusion as usual, do admit a finite axiomatization; see [4].

Although not full, $\mathcal{R}(X)$ is $*$-full, as we next show. In [31], Schein describes arbitrary idempotent relations on $X$ : they are quasi-orders possibly missing certain pairs $(x, x)$.

Lemma 3.3 For $X$ non-empty, $e \in E^{*}(\mathcal{R}(X))$ if and only if $e$ is an equivalence relation on $\operatorname{dom}(e)$.

Proof. Any equivalence relation on $Y \subseteq X$ is symmetric and transitive, so $e=e^{*}$ and $e^{2} \subseteq e$. Conversely, if $(x, y) \in e$ then $(y, x) \in e$, so $(x, x) \in e^{2} \subseteq e$, and since $(x, x),(x, y) \in e$, also $(x, y) \in e^{2}$. So $e^{2}=e$.

Conversely, if $e^{2}=e=e^{*}$, then $e$ is symmetric and transitive, and it follows easily that $e$ is an equivalence relation on $\operatorname{dom}(e)$.

Lemma 3.4 For $e, f \in E^{*}(\mathcal{R}(X)), e \leq f$ if and only if each e-class in dom(e) is a union of $f$-classes.

Proof. Suppose $e \leq f$. So $e=e f=f e$. If $x \in \operatorname{dom}(e)$ then $(x, x) \in e$ since $e$ is an equivalence relation on its domain. Hence there is $y$ such that $(x, y) \in e$ and $(y, x) \in f$. So $(x, y) \in f$ and so $x \in \operatorname{dom}(f)$. So $\operatorname{dom}(e) \subseteq \operatorname{dom}(f)$. If $(x, x) \in e$ and $(x, y) \in f$ then because $e=e f,(x, y) \in e$. So each $e$-class is a union of $f$-classes.

On the other hand, suppose $e, f$ are symmetric and transitive, and every e-class is a union of $f$-classes. If $(x, y) \in e$, then $(y, y) \in f$, so $(x, y) \in e f$, and so $e \subseteq e f$. Conversely if $(x, y) \in e f$ then $(x, u) \in e$ and $(u, y) \in f$ for some $u$, so as $(u, u) \in e$ and the $e$-class containing $u$ is a union of $f$-classes, we have that $(u, y) \in e$, and so $(x, y) \in e$ by transitivity, and so $e f \subseteq e$. Hence $e=e f=f e$, and $e \leq f$.

For $\rho \in \mathcal{R}(X)$, we define the kernel of $\rho$ in the obvious way:

$$
\operatorname{ker}(\rho)=\{(x, y) \mid(x, z) \in \rho \text { if and only if }(y, z) \in \rho \text {, for all } z \in X\} .
$$

Theorem 3.5 The involuted semigroup $\mathcal{R}(X)$ is $*$-full, with

$$
D(\rho)=\operatorname{ker}(\rho) \cap(\operatorname{dom}(\rho) \times \operatorname{dom}(\rho)) .
$$

Proof. If $D(\rho)$ is defined as above, it is easy to see that $D(\rho) \rho=\rho$, for all $\rho \in \mathcal{R}(X)$.

Suppose $\rho \in \mathcal{R}(X)$, and that $e \rho=\rho$ with $e \in E^{*}(\mathcal{R}(X))$. If $x \in \operatorname{dom}(\rho)$ then necessarily $x \in \operatorname{dom}(e)$, and so $(x, x) \in e$. So $\operatorname{dom}(D(\rho)) \subseteq \operatorname{dom}(e)$. If $(x, y) \in e$ with $x \in \operatorname{dom}(D(\rho))$ then $\rho$ must be such that $\rho(x)=\rho(y) \neq \emptyset$ as $x \in \operatorname{dom}(D(\rho))$, so $y \in \operatorname{dom}(D(\rho))$ and of course $(x, y) \in D(\rho)$ by definition. So each $D(\rho)$-class in $\operatorname{dom}(D(\rho))$ is a union of $e$-classes, and so $D(\rho) \leq e$ by Lemma 3.4.

The $*$-full D-semigroup $\mathcal{R}(X)$ does not generally satisfy even normality, as the following example shows. Let $X=\{a, b, c\}, s=\{(a, a),(b, b),(c, a),(c, b)\}$, with $e$ the (total) equivalence relation giving the partition $\{\{a, b\},\{c\}\}$ of $X$. Then

$$
e s=\{a, b, c\} \times\{a, b\}, D(e s)=\{\{a, b, c\}\},
$$


whereas $D(s)=\{\{a\},\{b\},\{c\}\}$ and so $e D(s)=e$. So $D(e D(s))=D(e)=e \neq D(e s)$.

A related D-semigroup structure on $\mathcal{R}(X)$ is obtained by letting $D(\mathcal{R}(X))$ consist of all equivalence relations on $X$, and then for $a \in \mathcal{R}(X), D(a)$ is the kernel of $a$ as defined above. This also gives a non-normal D-semigroup structure on $\mathcal{R}(X)$.

We next show that involuted D-semigroups are in a certain sense generic: all DRsemigroups embed in involuted D-semigroups.

If $S$ is a D-semigroup, then $S^{o p}$ is the R-semigroup which is the opposite semigroup to $S$ equipped with the obvious R-semigroup structure; similarly if $S$ is a DR-semigroup, then $S^{o p}$ is the opposite semigroup to $S$ equipped with the obvious DR-semigroup structure (obtained by interchanging $D, R$ ).

If $S$ is a semigroup, define $S * S$ to be $S \times S^{o p}$ equipped with involution as follows: $(s, t)^{*}=(t, s)$ for all $s, t \in S$. If $S$ is a DR-semigroup, then $S * S$ is a DR-semigroup since it is a direct product of two DR-semigroups, and indeed is involuted because for any $(e, f) \in D(S * S), D\left((e, f)^{*}\right)=D((f, e))=(D(f), R(e))=(f, e)=(e, f)^{*}$. Moreover if $S$ has a zero element then $S$ embeds in $S * S$ as a subsemigroup, a copy being $\{(x, 0) \mid x \in S\}$.

Proposition 3.6 Let $S$ be a D-semigroup (resp. DR-semigroup). If $S^{0}=S \cup\{0\}$ is the usual extension of $S$ by the inclusion of a zero element 0 not in $S$, then defining $D(0)=0$ makes $S^{0}$ a D-semigroup, in which $D\left(S^{0}\right)=D(S) \cup\{0\}$ (and define $R(0)=0$ in the DR-semigroup case). Moreover $S$ is a subalgebra (subsemigroup closed under $D$ ) of $S^{0}$.

It is easy to check that under the above construction, the congruence conditions and D-semiadequacy are passed from $S$ to $S^{0}$.

Proposition 3.7 Let $S$ be a DR-semigroup. Then $S$ embeds as a DR-semigroup in an involuted D-semigroup $T$, which satisfies the congruence (resp. normal, D-semiadequate, DR-abundant) conditions if $S$ does.

Proof. Assume that $S$ has a zero element 0 with $D(0)=R(0)=0$, the general result following from Proposition 3.6. $S * S$ has as sub-DR-semigroup $S_{1}=\{(x, 0) \mid x \in S\}$, which is easily seen to be isomorphic to $S$ as a DR-semigroup.

That the congruence, D-semiadequate, normal and DR-abundant properties are inherited follows because each of these properties is definable equationally.

The same construction allows us to reverse the process, and show that involuted Dsemigroups can themselves all be embedded in those of the form $S * S$ where $S$ is a DRsemigroup.

Corollary 3.8 Let $S$ be an involuted D-semigroup. Then $S$ embeds in the involuted $D$ semigroup $S * S$ (where $S$ is viewed simply as a DR-semigroup), which satisfies the congruence (resp. normal, D-semiadequate, DR-abundant) conditions if $S$ does.

Proof. Again assume that $S$ has a zero element 0, the general result following from Proposition 3.6 (slightly modified to account for the presence of the involution). Define 
$f: S \rightarrow S * S$ by setting $f(a)=\left(a, a^{*}\right)$; then it is easily checked (and is presumably well-known) that $f$ is an involuted semigroup embedding. Moreover, for $a \in S, D(f(a))=$ $D\left(\left(a, a^{*}\right)\right)=\left(D(a), R\left(a^{*}\right)\right)=\left(D(a), D(a)^{*}\right)=f(D(a))$, so $f$ is an involuted D-semigroup embedding. It follows from the previous result that the left congruence condition, as well as the normal and D-semiadequate properties, are passed from $S$ to $S * S$.

As in [8], a Baer *-semigroup is an involuted semigroup $S$ together with a particular choice of focal ideal $K$ of $S$ with the property that for every $a \in S$, the left ideal ( $a$ : $K)=\{y \in S \mid y a \in K\}$ is generated by a projection (a symmetric idempotent) $a^{\prime}$. (In fact this is a left-sided presentation of the notion considered in [8], obtained by reversing all products.) Baer $*$-semigroups include as special cases the multiplicative $*$-semigroups of Rickart *-rings, where one sets $K=\{0\}$. As noted in [8], $a^{\prime \prime} a=a$. Since for all $y \in S$, $y a \in K$ if and only if $y=y a^{\prime}$, it follows from $a^{\prime} a^{\prime}=a^{\prime}$ that $a^{\prime} a \in K$, so $a^{\prime} a b \in K$ for all $b$, so since also $z(a b) \in K$ if and only if $z(a b)^{\prime}=z$, we obtain $a^{\prime}(a b)^{\prime}=a^{\prime}$, so $a^{\prime} \leq(a b)^{\prime}$, and then it follows from one of the properties obeserved in [8] that $(a b)^{\prime \prime} \leq a^{\prime \prime}$. So setting $D(a)=a^{\prime \prime}$ for all $a \in S, S$ becomes a (symmetric involuted) D-semigroup. This generalises the previously observed fact for Rickart $*$-rings. Baer $*$-semigroups are not generally $*$-full.

\section{D-rings}

The example of Rickart *-rings shows that there are important D-semigroups arising as the multiplicative semigroups of rings. In such cases, the ring structure can shed light on the Dsemigroup structure. A number of the properties of D-semigroups have alternative simpler forms within the semigroups of rings, and often more can be said about the collection of domain elements.

If $A$ is a ring, we say it is a $D$-ring if it is multiplicatively a D-semigroup. All the defined properties for D-semigroups are assumed to pass up to D-rings in the obvious way. D-rings generalise the closure rings of [11], discussed earlier.

A D-ring which is an involuted ring for which the semigroup part is an involuted Dsemigroup is called an involuted D-ring. Similarly, a symmetric involuted D-ring $A$ satisfies $D(a)^{*}=D(a)$ for all $a \in A$. As for D-semigroups, an involuted D-ring is a DR-ring in which $R(a)=D\left(a^{*}\right)^{*}$ for all $a$.

\subsection{D-abundant D-rings}

If $D(0)=0$, the left congruence condition has an alternative form in rings.

Proposition 4.1 Let $A$ be a D-ring. The following conditions are equivalent.

1. A satisfies the left congruence condition and $D(0)=0$.

2. For all $a, b \in A$, if $a b=0$ then $a D(b)=0$.

Proof. $\quad(1) \Rightarrow(2)$. If $a b=0$, then $0=D(0)=D(a b)=D(a D(b))$, so $a D(b)=0$.

$(2) \Rightarrow(1)$. $D(0) 0=0$, so $D(0)=D(0) D(0)=0$. As noted previously, for all $a, b \in A$, $D(a b) \leq D(a D(b))$, so $D(a D(b)) a b=a b=D(a b) a b$, so $(D(a D(b))-D(a b)) a b=0$, and so 
by (2), we may infer that $(D(a D(b))-D(a b)) a D(b)=0$, and so $a D(b)=D(a b) a D(b)$, so $D(a D(b)) \leq D(a b)$. So the left congruence condition holds.

There is a converse of Proposition 2.2 in the ring case, at least when $D(0)=0$.

Proposition 4.2 Suppose $A$ is a D-ring. Then $A$ is D-abundant if and only if it satisfies the left congruence condition and $D(0)=0$.

Proof. Let $A$ satisfy the left congruence condition and $D(0)=0$, with $a \in A$. If $x a=y a$ where $x, y \in A$, then $(x-y) a=0$ so $(x-y) D(a)=0$ by Proposition 4.1. and so $x D(a)=y D(a)$. If $x a=a$ for some $x \in A$ then $(x-D(a)) a=0$, so $(x-D(a)) D(a)=0$, and so $x D(a)=D(a)$. So by Proposition 2.2, $A$ is D-abundant.

Conversely, if $A$ is D-abundant then it satisfies the left congruence condition by Proposition 2.2. By the same result, because $0 \cdot 0=1 \cdot 0$ in the multiplicative semigroup $A^{1}$, $0 D(0)=1 D(0)$, that is, $D(0)=0$.

Corollary 4.3 Suppose $A$ is a D-ring. Then $A$ is D-abundant if and only if $a b=0$ implies $a D(b)=0$ for all $a, b \in A$.

There are obvious definitions for "R-abundant" and "DR-abundant", and corresponding variants of the above (and forthcoming) results.

The closure rings considered in [11] are D-semiadequate D-rings. Examples that are not D-semiadequate are furnished by rings of operators. A (left) Rickart ring is a ring $R$ in which the left annihilator $(0: a)=\{r \in R \mid r a=0\}$ of each $a \in R$ is generated as a left ideal by an idempotent (see [25]). Examples include all Baer rings. For many of the major results, [3] is a convenient reference.

Theorem 4.4 Let $A$ be a D-ring with identity. Then $A$ is D-abundant if and only if $A$ is a Rickart ring in which $(0: a)=A(1-D(a))$ for all $a \in A$.

Proof. Let $e \in D(A)$. For $x \in A, x e=0$ if and only if $x(1-e)=x$, if and only if $x=y(1-e)$ for some $y \in A$ as is easily seen. So $(0: e)=A(1-e)$.

Suppose $A$ is D-abundant. For each $a \in A, x a=0$ if and only if $x D(a)=0$, if and only if $x(1-D(a))=x-x D(a)=x$. So $(0: a)=\{x \in A \mid x a=0\}=(0: D(a))=A(1-D(a))$.

Conversely, suppose $A$ is a Rickart ring, with $(0: a)=A(1-D(a))$ for all $a \in A$. So $(0: a)=(0: D(a))$ for all $a \in A$. So if $x a=0$ for $x, a \in A$, then $x D(a)=0$, so $A$ is D-abundant by Corollary 4.3 .

An arbitrary Rickart ring $A$ will not be a D-ring in any obvious way since in general there are many candidates for the idempotent $e$ that generates $(0: a), a \in A$. This is remedied by requiring that the idempotent be symmetric with respect to an involution, yielding the notion of a Rickart $*$-ring, referred to in the Introduction.

There is a characterisation of D-abundant D-rings with identity that represents a generalisation of the definition of Rickart *-rings. Let $A$ be a ring with identity. We say $E \subseteq E(A)$ is complement-closed if $1-e \in E$ whenever $e \in E$. Thus $E(A)$ is complement-closed, and so is $E^{*}(A)$ if $A$ is involuted. 
Proposition 4.5 Let $A$ be a ring with identity, with $U \subseteq E(A)$ complement-closed. The following are equivalent.

1. $A$ is a D-abundant $D$-ring in which $D(A)=U$.

2. $U$ is left-reduced, and for all $a \in A$, there exists $e \in U$ for which $(0: a)=A e$.

Proof. Suppose (1) holds. Then by Theorem 4.4, $A$ is a Rickart ring in which for all $a \in A,(0: a)=A(1-D(a))=A e$ for some $e \in D(A)=U$ since $e=1-D(a) \in D(A)$. Of course $U$ is left-reduced. So (2) holds.

Conversely, suppose (2) holds. Then $A$ is a Rickart ring. Pick $a \in A$, and suppose $(0: a)=A e$ where $e \in U$. Then $e=e^{2} \in A e$, so $e a=0$, and so $e^{\prime} a=a$ where $e^{\prime}=1-e \in U$. If $f a=a$ for some $f \in U$ then $(1-f) a=0$, so $1-f \in(0: a)=A e$, and so $1-f=(1-f) e$. Hence $f(1-e)=f-f e=1-e$, so because $U$ is left-reduced, $1-e \leq f$. Hence setting $D(a)=1-e$ makes $A$ a D-ring in which $D(A)=U$. So the Rickart ring $A$ satisfies $(0: a)=A((1-D(a))$ for all $a \in A$ as just shown. So $A$ is D-abundant by Theorem 4.4 .

Every Rickart $*$-ring $A$ has an identity element which is a projection, since $A=(0: 0)$, so $A=A e$ for some projection $e$, which is therefore a right identity. But for all $a \in A$, $a^{*}=(a e)^{*}=e^{*} a^{*}=e a^{*}$, so $e$ is also a left identity (since every $b \in A$ equals $\left.\left(b^{*}\right)^{*}\right)$, and hence is an identity element; call it 1 .

Corollary 4.6 Let $A$ be an involuted ring with identity. The following are equivalent.

1. A is a D-abundant symmetric involuted D-ring.

2. A is a Rickart *-ring.

Proof. Assume (1). By Lemma 3.2, $D(A)=E^{*}(A)$, which is trivially complement-closed and so by Proposition 4.5, $A$ is a a Rickart *-ring, proving (2).

Conversely, if (2) holds, then $A$ with $U=E^{*}(A)$ is obviously complement-closed, and clearly satisfies condition (2) in Proposition 4.5, so $A$ is a D-abundant D-ring, and evidently symmetric involuted.

It follows that the class of Rickart *-rings is exactly the class of (reducts of) D-abundant symmetric involuted D-rings with identity, and the correspondence is one-to-one. The latter class is a variety of involuted D-rings, hence closed under the usual operators of taking subalgebras, homomorphic images and direct products.

The D-ring point of view gives rise to an obvious generalisation of Rickart $*$-rings to the case of rings without identity. For example, the ring of bounded linear operators on a Hilbert space that have finite-dimensional images constitutes a sub-involuted D-ring of the Rickart *-ring of all bounded linear operators, but has no identity element and so is not itself a Rickart $*$-ring.

Similarly, there are natural examples without involution. The Rickart $*$-ring $M_{3}(\mathbb{R})$ of $3 \times 3$ matrices with real entries (in which involution is transpose) contains as a sub-D-ring $A$ consisting of those matrices having third row all zeros. Note that, since $D(a)^{*}=D(a)$ for all $a \in M_{3}(\mathbb{R}), D(A)$ consists of the symmetric idempotents in $A$, and these have all zeros 
in the third column as well. It is straightforward to see that, with this $D, A$ is a sub-D-ring of $M_{3}(\mathbb{R})$. Clearly $A$ is not a $*$-ring under transpose.

This line of argument generalises to the right annihilator of any element in a D-abundant D-ring (such as a Rickart *-ring), which constitutes a sub-D-ring. In the case just considered, one element of $M_{3}(\mathbb{R})$ having right annihilator $A$ is the matrix with a " 1 " in the bottom right position and which is zero elsewhere.) In general, the right annihilator of an element in a Rickart $*$-ring is not closed under $*$.

All the examples just mentioned are at least D-abundant D-rings. It is therefore of interest to generalise known facts about Rickart *-rings at least to D-abundant D-rings, or perhaps even further. Indeed, Corollary 2.4 for D-abundant D-semigroups is a generalisation of 3.8 in [3]. We obtain further such generalisations in what follows.

\subsection{D-semiadequate D-rings}

There are non-trivial examples of D-semiadequate DR-semigroups satisfying both congruence conditions, such as $\mathcal{R}(X)$, with $D(\mathcal{R}(X))$ defined to be the subsets of the diagonal relation. But if the congruence conditions hold on a D-semiadequate DR-ring, it is of a quite restricted form (at least if $D(0)=R(0)=0$, so that it is DR-abundant).

A ring is said to be abelian if $E(A)=C(A)$. By analogy, we say the D-ring $A$ is $D$ abelian if $D(A) \subseteq C(A)$. Obviously, D-abelian D-rings are D-semiadequate. By Corollary 2.4, if the D-abundant D-ring $A$ is D-abelian, then $D(A)=C(A)$.

Evidently a D-abelian D-ring $A$ is a DR-ring: $R(a)=D(a)$ for all $a \in A$. (In [11], this notion was considered in a more restricted way in the setting of closure rings: there, a closure ring was said to be central if $D(A)$ equals the set of all central idempotents of $A$. Here, we only require containment.)

Theorem 4.7 Let $A$ be a DR-abundant DR-ring. The following are equivalent.

1. A is D-semiadequate.

2. $A$ is abelian.

3. $D(a)=R(a)$ for all $a \in A$.

When any of these holds, $A$ is full.

Proof. Assume (1). Let $e \in E(A)$. From Lemma 2.3 and its right dual, $D(e)=e D(e)$ and $R(e)=R(e) e$, so

$$
D(e) R(e)=D(e) R(e) e=R(e) D(e) e=R(e) e=R(e)
$$

so $R(e) \leq D(e)$, and similarly $D(e) \leq R(e)$, so $D(e)=R(e)$. Hence $e=D(e) e=R(e) e=$ $R(e)$, so $e \in D(A)$. So $A$ is D-full. But by a result on page 10 of [18], a ring is abelian if and only if all of its idempotents commute with each other. So (2) now follows. That (2) implies (1) is immediate.

That (2) implies (3) is immediate. If (3) holds, let $e \in E(A)$ and $a \in A$, and let $r=e a-e a e$. Then it is easily checked that $r^{2}=0$, so $r D(r)=0$, and so $R(r) D(r)=0$, 
so $D(r)=D(r)^{2}=0$ by assumption. Hence $r=D(r) r=0$. So ea =eae. By symmetry, $a e=e a e$ also. So $e a=a e$. So (2) holds.

Theorem 4.7 is a generalisation and extension of 8.10 and 8.12 in [3] (the former first appearing in [18]).

\subsection{Involuted D-rings}

We briefly return to involuted D-rings in order to mention some easy extensions to rings of earlier results for involuted D-semigroups. If $A$ is a ring, define $T=A * A$ to be the direct product of the ring $A$ and its opposite ring, equipped with the same involution as in the semigroup case discussed in Section 3. This makes $T$ an involuted ring as well as a $D$-semigroup. The involution operation on $A * A$ is called the exchange involution; see [15] for example.

We have the following easy variant of Proposition 3.7.

Proposition 4.8 Let $A$ be a DR-ring. Then $A$ embeds as a DR-ring in an involuted D-ring $T$, which is D-abundant (resp. D-semiadequate) if $A$ is.

The mapping $f$ of Corollary 3.8 is easily seen to be an involuted ring homomorphism, and we obtain the following.

Corollary 4.9 Let $A$ be an involuted D-ring. Then $A$ embeds in the involuted $D$-ring $A * A$ (where $A$ is viewed simply as a DR-ring), which is D-abundant (resp. D-semiadequate) if $A$ is.

\subsection{Strong D-rings}

Recall that a strong D-semigroup is one for which for all $a \in S$ there exists $b \in S$ such that $a b=D(a)$. We say a D-ring is strong if its multiplicative D-semigroup is strong. Strongness trivially implies regularity, and by Proposition 2.6 also implies the left congruence condition, although the converse was shown to fail for D-semigroups. For rings, the converse does hold (at least when $D(0)=0$ ). The next result is a generalisation of (most of) Theorem $4.5 \mathrm{in}$ [28]; see also Proposition 1.13 in [3].

Theorem 4.10 Let $A$ be a D-ring. The following are equivalent.

1. A is strong.

2. $A$ is regular and D-abundant.

Proof. $\quad(1) \Rightarrow(2)$. It remains to show $D(0)=0$. But $0 b=D(0)$ for some $b \in A$, so $D(0)=0$.

$(2) \Rightarrow(1)$. For all $a \in A$, there is $b \in A$ such that $a b a=a$. So $(a b-D(a)) a=0$, and so $(a b-D(a)) D(a)=0$ by Proposition 4.1. So $a(b D(a))=D(a)$, and so $A$ is strong. 


\subsection{Some matrix ring examples}

Examination of some $2 \times 2$ matrix rings illustrates a wide range of possible behaviours.

If $A$ is a ring with identity, we say $M \in M_{n}(A)$ is $0 / 1$-diagonal if its only non-zero entries are on the main diagonal and equal 1. For $M \in M_{n}(A)$, define $D(M)$ to be the smallest 0/1-diagonal matrix for which $D(M) M=M$, meaning the one with fewest non-zero entries (which will be the smallest such matrix under the standard order on 0/1-diagonal matrices). This exists: multiply together all 0/1-diagonal matrices $F$ for which $F M=M$ and the result is $D(M)$. We call this D-ring structure on $M_{n}(A) 0 / 1$-diagonal; clearly it is D-semiadequate.

Proposition 4.11 If $A$ is non-trivial, the 0/1-diagonal D-ring structure on $M_{n}(A)$ is not D-abundant.

Proof. If

$$
M=\left(\begin{array}{ll}
1 & 1 \\
0 & 0
\end{array}\right), N=\left(\begin{array}{cc}
1 & 1 \\
-1 & -1
\end{array}\right)
$$

then $M N=0$, while $D(N)=I_{2}$, the identity matrix, and so $M \cdot D(N)=M \neq 0$, so by Corollary 4.3 the D-abundant condition is not satisfied.

The 0/1-diagonal D-ring structure on $M_{n}(A)$ is symmetric involuted under transpose, hence two-sided, with $R(M)$ having an obvious definition in terms of the columns of $M$ rather than its rows.

Note that $M_{n}(\mathbb{R})$ is isomorphic to the ring of (bounded) linear operators on the Hilbert space $\mathbb{R}^{n}$, hence is a Rickart $*$-ring (where involution is transpose), hence $*$-full and Dabundant. It is of interest to determine those rings $A$ and those values of $n$ for which $M_{n}(A)$ is $*$-full (where the involution is transposition), and whether it is D-abundant.

If $A$ is an integral domain and $n=2, E^{*}\left(M_{2}(A)\right)$ will always at least include the set of 0/1-diagonal matrices over $A$ :

$$
0=\left(\begin{array}{ll}
0 & 0 \\
0 & 0
\end{array}\right), e_{1}=\left(\begin{array}{ll}
1 & 0 \\
0 & 0
\end{array}\right), e_{2}=\left(\begin{array}{ll}
0 & 0 \\
0 & 1
\end{array}\right) \text { and } I_{2}=\left(\begin{array}{ll}
1 & 0 \\
0 & 1
\end{array}\right) .
$$

The conditions on the symmetric matrix $M=\left(\begin{array}{cc}a & b \\ b & c\end{array}\right) \in M_{2}(A)$ that ensure it is idempotent are

$$
a^{2}+b^{2}=a, a b+b c=b, b^{2}+c^{2}=c .
$$

If $E^{*}\left(M_{n}(A)\right)$ is to contain any non-diagonal idempotents, then it must be possible for $b$ to be non-zero. In that case, the second equation gives $a+c=1$, and it is easy to see that the first and third equations are equivalent to $a(1-a)=b^{2}$. For many choices of $A$ (such as the integers $\mathbb{Z})$, this forces $b=0$, a contradiction, and so for such $A, E^{*}\left(M_{2}(A)\right)$ consists of the 0/1-diagonal matrices and we obtain the 0/1-diagonal D-ring structure on $M_{2}(A)$, which is not D-abundant.

Over $\mathbb{Z}_{2}$, the equation $a(1-a)=b^{2}$ has no non-zero solutions for $b$, so $M_{2}\left(\mathbb{Z}_{2}\right)$ is *-full, and again we recover the 0/1-diagonal D-ring structure. But over $\mathbb{Z}_{3}$, we obtain 
non-zero solutions to $a(1-a)=b^{2}$, and it is easy enough to discover that $E^{*}\left(M_{2}\left(\mathbb{Z}_{3}\right)\right)=$ $\left\{0, e_{1}, e_{2}, I_{2}, f_{1}, f_{2}\right\}$, where

$$
f_{1}=\left(\begin{array}{ll}
2 & 2 \\
2 & 2
\end{array}\right), f_{2}=\left(\begin{array}{ll}
2 & 1 \\
1 & 2
\end{array}\right)
$$

None of $e_{1}, e_{2}, f_{1}, f_{2}$ is comparable under the standard order on $E^{*}\left(M_{2}\left(\mathbb{Z}_{3}\right)\right)$, and 0,1 are bottom and top elements respectively. If $e \in E^{*}\left(M_{2}\left(\mathbb{Z}_{3}\right)\right)$ and $M=\left(\begin{array}{ll}a & b \\ c & d\end{array}\right) \in M_{2}\left(\mathbb{Z}_{3}\right)$, $e M=M$ implies $e=I_{2}$ if and only if $M$ is non-singular, and $e M=M$ for precisely one other choice of $e$ otherwise:

- for $M=0, e=0$;

- for $M=\left(\begin{array}{ll}a & b \\ 0 & 0\end{array}\right)$ where not both $a, b$ are zero, $e=e_{1}$;

- for $M=\left(\begin{array}{ll}0 & 0 \\ c & d\end{array}\right)$ where not both $b, c$ are zero, $e=e_{2}$;

- for $M=\left(\begin{array}{ll}a & b \\ a & b\end{array}\right)$ where not both $a, b$ are zero, $e=f_{1}$;

- for $M=\left(\begin{array}{cc}a & b \\ -a & -b\end{array}\right)$ where not both $a, b$ are zero, $e=f_{2}$.

It follows easily that $M_{2}\left(\mathbb{Z}_{3}\right)$ is a $*$-full D-ring with $D(M)=1$ if $M \in M_{2}\left(\mathbb{Z}_{3}\right)$ is nonsingular, $D(0)=0$, and otherwise $D(M)$ is determined as above (noting that a singular matrix must have one of the forms considered above).

Suppose $M N=0$ for $M, N \in M_{2}\left(\mathbb{Z}_{3}\right)$. If $M=0$ or $N=0$, it is immediate that $M \cdot D(N)=0$. So suppose $M, N \neq 0$; then both must be singular. If $N=\left(\begin{array}{cc}a & b \\ a & b\end{array}\right)$, so that $D(N)=f_{1}$, and $M=\left(\begin{array}{ll}p & q \\ r & s\end{array}\right)$, it is easy enough to show that $p+q=r+s=0$, and so $M \cdot D(N)=0$. The various other cases may be similarly dealt with, establishing that indeed $M_{2}\left(\mathbb{Z}_{3}\right)$ is D-abundant (but not D-semiadequate by Theorem 4.7).

Arguing along similar lines, one sees that

$$
E^{*}\left(M_{2}\left(\mathbb{Z}_{5}\right)\right)=\left\{0, e_{1}, e_{2}, I_{2}, g_{1}, g_{2}\right\}
$$

where

$$
g_{1}=\left(\begin{array}{ll}
3 & 3 \\
3 & 3
\end{array}\right), g_{2}=\left(\begin{array}{ll}
3 & 2 \\
2 & 3
\end{array}\right)
$$

that $M_{2}\left(\mathbb{Z}_{5}\right)$ ) is $*$-full (in a very similar way to the $\mathbb{Z}_{3}$ case), but not D-abundant, since

$$
\left(\begin{array}{ll}
1 & 2 \\
1 & 2
\end{array}\right)\left(\begin{array}{ll}
0 & 1 \\
0 & 2
\end{array}\right)=0, \text { yet } D\left(\left(\begin{array}{ll}
0 & 1 \\
0 & 2
\end{array}\right)\right)=g_{2} \text { and }\left(\begin{array}{ll}
1 & 2 \\
1 & 2
\end{array}\right)\left(\begin{array}{ll}
3 & 2 \\
2 & 3
\end{array}\right) \neq 0,
$$

nor D-semiadequate since for example $e_{1} g_{1} \neq g_{1} e_{1}$. 
We conjecture that $M_{2}(\mathbb{F})$ is a $*$-full D-ring for any field $\mathbb{F}$. By contrast, it is not hard to show that $E^{*}\left(M_{3}\left(\mathbb{Z}_{2}\right)\right)$ consists of the eight 0/1-diagonal matrices, together with

$$
f=\left(\begin{array}{lll}
1 & 1 & 1 \\
1 & 1 & 1 \\
1 & 1 & 1
\end{array}\right), g=\left(\begin{array}{lll}
0 & 1 & 1 \\
1 & 0 & 1 \\
1 & 1 & 0
\end{array}\right)
$$

But letting $e=\left(\begin{array}{lll}1 & 0 & 0 \\ 0 & 1 & 0 \\ 0 & 0 & 0\end{array}\right)$ and $M=\left(\begin{array}{lll}1 & 1 & 1 \\ 1 & 1 & 1 \\ 0 & 0 & 0\end{array}\right)$, it is easy to verify that $e M=f M$, yet there is no non-zero element of $E^{*}\left(M_{3}\left(\mathbb{Z}_{2}\right)\right)$ below both $e, f$ under the standard order, so $M_{3}\left(\mathbb{Z}_{2}\right)$ is not $*$-full.

Returning to $M_{2}(A)$ but with $A=\mathbb{Z}_{4}$,

$$
E^{*}\left(M_{2}\left(\mathbb{Z}_{4}\right)\right)=\left\{0, e_{1}, e_{2}, I_{2}, f, g\right\},
$$

where $f=\left(\begin{array}{ll}1 & 2 \\ 2 & 0\end{array}\right)$ and $g=\left(\begin{array}{ll}0 & 2 \\ 2 & 1\end{array}\right)$. If $M=\left(\begin{array}{ll}2 & 2 \\ 0 & 0\end{array}\right)$, then $e_{1} M=f M=M$, but no smaller element $h$ of $E^{*}\left(M_{2}\left(\mathbb{Z}_{4}\right)\right)$ satisfies $h M=M$, so $M_{2}\left(\mathbb{Z}_{4}\right)$ is not $*$-full. $M_{2}\left(\mathbb{Z}_{4}\right)$ can however be made into a non-D-abundant symmetric involuted D-ring in which $D\left(M_{2}\left(\mathbb{Z}_{4}\right)\right)=$ $\left\{0, e_{1}, I_{2}, g\right\}$.

\section{$5 \quad$ Strong normality in D-rings}

The normality property did not feature greatly in our work on D-semigroups, but does arise naturally in the setting of D-rings.

\subsection{Defining strong normality}

If $A$ is a D-ring with identity, we say it is complement-closed if $D(A)$ is complementclosed. The complement-closed D-rings with identity form a variety of D-rings with identity, specified by the law $D(1-D(a))=1-D(a)$. Every full D-ring with identity and every $*$-full involuted D-ring with identity is complement-closed; in particular, every Rickart $*$-ring is complement-closed, as are the D-rings described in the statement of Proposition 4.5. By contrast, defining $D(a)=1$ for all $a \in A$ makes any non-trivial ring with identity $A$ into a D-ring which is not complement-closed.

We say the D-ring $A$ is strongly normal if, for all $a \in A$ and $e \in D(A), D(a-e a) e=0$.

Proposition 5.1 The D-ring with identity $A$ is complement-closed if and only if it is strongly normal.

Proof. Suppose $A$ is complement-closed. Then for $e \in D(A)$ and $a \in A$,

$$
D((1-e) a)(1-e)=D((1-e) a) D(1-e)=D((1-e) a),
$$

so $D(a-e a) e=D((1-e) a) e=0$. 
Conversely, suppose that for all $a \in A$ and $e \in D(A), D(a-e a) e=0$. Then for all $e \in D(A)$, setting $a=1$ gives $D(1-e) e=0$. So

$$
1-e=D(1-e)(1-e)=D(1-e)-D(1-e) e=D(1-e),
$$

and so $A$ is complement-closed.

Strong normality therefore provides a convenient generalisation of the complementclosed notion to D-rings without identity. It has a useful equivalent form.

We say the D-ring $A$ is quasinormal if for all $a \in A$ and $e \in D(A)$, ea $=0 \Rightarrow e D(a)=0$. Note that since $D(0) 0=0$, by quasinormality, $D(0) D(0)=0$, and so $D(0)=0$.

The quasinormality condition has an equivalent equational form.

Proposition 5.2 The D-ring $A$ is quasinormal if and only if it satisfies the law eD(a$e a)=0$ for all $a \in A$ and $e \in D(A)$.

Proof. Suppose $a \in A, e \in D(A)$. If $A$ is quasinormal, then because $e(a-e a)=0$, we obtain $e D(a-e a)=0$. Conversely if the law is satisfied and $e a=0$ then $0=e D(a-e a)=$ $e D(a)$, so $A$ is quasinormal.

Proposition 5.3 If the D-ring $A$ is normal and $D(0)=0$ then it is quasinormal.

Proof. Suppose $A$ is normal and $D(0)=0$. If $a \in A$ and $e \in D(A)$ are such that $e a=0$, then $0=D(0)=D(e a)=D(e D(a))$, so $e D(a)=0$.

Lemma 5.4 The D-ring $A$ is strongly normal if and only if it is quasinormal and for all $e, f \in D(A)$, ef $=0$ implies $f e=0$. In that case, $A$ is reduced in the sense introduced in the Introduction for D-semigroups.

Proof. Suppose $A$ is quasinormal and satisfies $e f=0$ implies $f e=0$ for all $e, f \in D(A)$. Then $e D(a-e a)=0$, and so $D(a-e a) e=0$.

Conversely, suppose $A$ is strongly normal. If ef $=0$ for some $e, f \in D(A)$, then $0=D(f-e f) e=D(f) e=f e$. So $e D(a-e a)=0$ since $D(a-e a) e=0$, so $A$ is quasinormal by Proposition 5.2.

Finally, suppose $A$ is strongly normal (hence quasinormal and satisfies ef $=0$ implies $f e=0$ for all $e, f \in D(A))$. If $e f=e$ for $e, f \in D(A)$, then $e(f e-e)=e f e-e^{2}=e-e=0$, so $e D(f e-e)=0$, so $D(f e-e) e=0$. Also, $f(f e-e)=f^{2} e-f e=0$, so $f D(f e-e)=0$, and so $D(f e-e) f=0$. Hence

$$
f e-e=D(f e-e)(f e-e)=D(f e-e) f e-D(f e-e) e=0-0=0,
$$

and so $e=f e$, which shows that $A$ is right-reduced, hence reduced.

If $A$ is a symmetric involuted D-ring, then the implication $e f=0 \Rightarrow f e=0$ is obviously satisfied for $e, f \in D(A)$, so strong normality is equivalent to quasinormality. 
The quasinormality property is a proper generalisation of the D-abundant property in rings, as the example of $M_{n}(A)$ where $A$ is a ring with identity, equipped with its $0 / 1$ diagonal D-ring structure, shows: this is strongly normal since $D\left(M_{n}(A)\right)$ is complementclosed, but is not D-abundant by Proposition 4.11. It also follows that the D-ring structure on $M_{2}\left(\mathbb{Z}_{4}\right)$ given at the end of Section 4.5 is not D-abundant, since it cannot be strongly normal (as $M_{2}\left(\mathbb{Z}_{4}\right)$ is not complement-closed).

Lemma 5.5 Let $A$ be a strongly normal D-ring. Then $D(A)$ is closed under differences, in the sense that if $e, f \in D(A)$ with $f \leq e$, then $e-f \in D(A)$.

Proof. For all $e, f \in D(A)$, if $f \leq e$ then $f=e f=f e$, and $D(e-f e) f=0$ by strong normality. So

$$
e-f=D(e-f)(e-f)=D(e-e f) e-D(e-f e) f=D(e-e f),
$$

so $D(A)$ is closed under differences.

We can now characterise strong normality in terms of normality, justifying the name.

Proposition 5.6 The D-ring $A$ is strongly normal if and only if it is normal, satisfies $D(0)=0$, and ef $=0 \Rightarrow f e=0$ for all $e, f \in D(A)$.

Proof. Suppose the D-ring $A$ is strongly normal. Let $a \in A$ and $e \in D(A)$. Then, as for the left congruence condition, it is easy to see that $D(e a) \leq D(e D(a))$. So $f=$ $D(e D(a))-D(e a) \in D(A)$ by closure under differences. Now it is straightforward to check that $f e=f$ and that $f(e a)=0$, so $f a=0$, and so $f D(a)=0$ (note that $A$ is quasinormal by Lemma 5.4), so $f e D(a)=0$, and so $f D(e D(a))=0$, again by quasinormality, which when multiplied out gives that $D(e D(a))-D(e a) D(e D(a))=0$, and so by left-regularity, $D(e D(a)) \leq D(e a)$ as required for normality. By quasinormality, $D(0)=0$, and the last property follows from Lemma 5.4.

Conversely, suppose $A$ is normal and satisfies $D(0)=0$, and ef $=0$ implies $f e=0$ for all $e, f \in D(A)$. Then it is quasinormal by Proposition 5.3, hence strongly normal by Lemma 5.4.

Hence a symmetric involuted D-ring is strongly normal if and only if it is normal and satisfies $D(0)=0$.

In fact general complement-closed D-rings (strongly normal D-rings with identity) admit a characterisation having a similar flavour to the Rickart $*$-ring definition (c.f. also Proposition 4.5).

Proposition 5.7 Let $A$ be a ring with identity, with $U \subseteq E(A)$ complement-closed. Then $A$ is a complement-closed D-ring in which $D(A)=U$ if and only if $U$ is left-reduced and for all $a \in A$ there exists $e \in U$ for which $(0: a) \cap U=U$ e.

Proof. Suppose $A$ is a complement-closed D-ring, and let $U=D(A)$. So for all $a \in A$ and $f \in U, f a=0$ if and only if $f D(a)=0$ for all $f \in D(A)$ by quasinormality, if and only if $f(1-D(a))=f$, if and only if $f \in U(1-D(a))$. So $(0: a) \cap U=U e$ where $e=1-D(a) \in U$ by complement-closure. Finally, $U$ is left-reduced by Proposition 1.3. 
Conversely, suppose $U$ is left-reduced and for all $a \in A$ there exists $e \in U$ for which $(0: a) \cap U=U e$. Then for $e \in U, e \in U e$, so $e a=0$, and so $(1-e) a=a$, with $1-e \in U$. If also $f a=a$ for some $f \in U$ then $(1-f) a=0$, so $(1-f) \in U e$, and so $1-f=(1-f) e$, from which it follows that $f(1-e)=1-e$, and so since $U$ is left-reduced, $1-e \leq f$. So setting $D(a)=1-e$ makes $A$ a D-ring in which $D(A)=U$.

We next see that one of the most important properties of Rickart *-rings generalises to complement-closed D-rings, indeed to strongly normal D-rings generally: even the Dabundant property proves unnecessary.

\subsection{The structure of $D(A)$}

In a D-semiadequate D-semigroup $S$, the poset $D(S)$ is a semilattice under multiplication, but in a general D-semigroup $D(S)$ need not even be closed under mutiplication. $D(S)$ is a lattice under its standard order in a number of the non-D-semiadequate examples considered (for example in $S=\mathcal{R}(X)$ if $D(S)$ consists of all equivalence relations, since the standard order is simply the dual of the inclusion order in this case, as is easily checked), although the lattice operations cannot be expressed in the language of D-semigroups in general. Those D-semiadequate D-rings $A$ in which $D(A)$ is closed under the circle composition operation ○ are axiomatized in [11]; in such cases $D(A)$ is a distributive lattice under its standard order, in which multiplication is meet and $\circ$ is join.

In the non-D-semiadequate case, it is well-known that in a Rickart *-ring $A$ (that is, a D-abundant symmetric involuted D-ring $A$, by Corollary 4.6), $D(A)=E^{*}(A)$ is a lattice in which, for all $e, f \in D(A), e \vee f=f+D(e-f e)$ and $e \wedge f=e-D(e-e f)$ are join and meet respectively; this is essentially Lemma 5.3 of [17] (see also Proposition 1.15 of [3]), after noting that $R(a)=D\left(a^{*}\right)$. Indeed, $D(A)$ is an orthomodular lattice, with the orthocomplement of $e \in D(A)$ being $1-e$.

Here we seek the most general conditions on a D-ring $A$ which ensure that $D(A)$ is a lattice under these same purely D-ring operations. We shall show that strong normality of $A$ is the necessary and sufficient condition. In particular, the D-ring need not be D-abundant, involuted or even a DR-ring, so can be very far from a Rickart *-ring. It follows from Proposition 5.1 that for a D-ring with identity $A$, the required condition is complementclosure, which is also enough to ensure that $D(A)$ is orthomodular.

If $A$ is a D-ring, define $e \curlyvee f=f+D(e-f e)$ and $e \curlywedge f=e-D(e-e f)$ for all $e, f \in D(A)$. These need not be lattice operations on $D(A)$, which may not even be a lattice in general. However, if $D(A)$ is to be a lattice under these operations, $A$ must necessarily be strongly normal.

Proposition 5.8 Let $A$ be a D-ring. If the poset $D(A)$ is a lattice in which $\curlyvee$ is join and $\curlywedge$ is meet, then $A$ is strongly normal.

Proof. If $e \leq f$ in $D(A)$ then $e=f \curlywedge e=f-D(f-f e)=f-D(f-e)$, and so $D(f-e)=f-e$.

Suppose $e a=0$, for some $e \in D(A)$ and $a \in A$. Then $e \leq e \curlyvee D(a)$, so $e \curlyvee D(a)-e \in$ $D(A)$, and

$(e \curlyvee D(a)-e) a=(e \curlyvee D(a)-e) D(a) a=((e \curlyvee D(a)) D(a)-e D(a)) a=D(a) a-e a=a$, 
so $D(a) \leq e \curlyvee D(a)-e$, and so $(e \curlyvee D(a)-e) D(a)=D(a)$, and so $e D(a)=0$.

If $e f=0$ for some $e, f \in D(A)$ then $e+f=e+D(f-e f)=(f \curlyvee e) \in D(A)$. So $e+f=(e+f)^{2}=e+f+e f+f e=e+f+f e$, and so $f e=0$.

So by Lemma 5.4, $A$ is strongly normal.

We now prove sufficiency of strong normality.

Theorem 5.9 Let $A$ be a strongly normal D-ring. Then $D(A)$ is a lattice under its standard order, in which for all e, $f \in D(A)$, e $\curlywedge f \in D(A)$ is their meet and $e \curlyvee f$ is their join.

Proof. For $e, f \in D(A), D(e-e f) \leq e$, so $e-D(e-e f) \in D(A)$ by Lemma 5.5. Furthermore, $e(e-D(e-e f))=e-D(e-e f)$, so $e-D(e-e f) \leq e$, since $A$ is reduced by Lemma 5.4. Moreover,

$$
\begin{aligned}
(e-D(e-e f)) f & =e f-D(e-e f) e f \\
& =e f+D(e-e f)(e-e f)-D(e-e f) e \\
& =e f+(e-e f)-D(e-e f) \\
& =e-D(e-e f),
\end{aligned}
$$

and so $e-D(e-e f) \leq f$, again because $A$ is reduced.

Suppose $g \leq e$ and $g \leq f$. Then $g=g e=e g$ and $g=g f=f g$, so $g(e-D(e-e f))=$ $g-g D(e-e f)$. Also, $g(e-e f)=g e-g e f=g-g f=g-g=0$, so $g D(e-e f)=0$ by quasinormality, and so $g(e-D(e-e f))=g$, so $g \leq e-D(e-e f)$ because $A$ is reduced. So $(D(A), \curlywedge)$ is a semilattice in which $\curlywedge$ is meet with respect to the standard order on $D(A)$.

Suppose $e f=f e=0$ for some $e, f \in D(A)$. If $g \in D(A)$ satisfies $g(e+f)=e+f$ then postmultiplying by $e, f$ in turn gives $g e=e$ and $g f=f$, so $e, f \leq g$, so in particular $e, f \leq D(e+f)$. So by Lemma 5.5, $D(e+f)-e \in D(A)$ and $D(e+f)-f \in D(A)$. Also, $(D(e+f)-f) e=D(e+f) e=e=e D(e+f)=e(D(e+f)-f)$, so $e \leq D(e+f)-f$. So $D(e+f)-(e+f)=D(e+f)-f-e \in D(A)$. But $D(e+f)(D(e+f)-(e+f))=$ $D(e+f)-(e+f)$, so $D(e+f)-(e+f) \leq D(e+f)$, and so $D(e+f)-(D(e+f)-(e+f)) \in D(A)$, that is, $e+f \in D(A)$.

Again, for $e, f \in D(A), f(e-f e)=0$, so $f D(e-f e)=0$, and so $D(e-f e) f=0$. So from the above, $f+D(e-f e) \in D(A)$. We must show $f+D(e-f e)$ is the least upper bound of $e, f$. As we have just seen, $D(e-f e) f=0$, so

$$
\begin{aligned}
(f+D(e-f e)) e & =f e+D(e-f e) e \\
& =f e+D(e-f e)(e-f e)+D(e-f e) f e \\
& =f e+(e-f e)+0 \\
& =e,
\end{aligned}
$$

so $e \leq e \curlyvee f$. But $f D(e-f e)=0$, so $f(f+D(e-f e))=f+f D(e-f e)=f$, so $f \leq f+D(e-f e)$ also. So $e \curlyvee f$ is an upper bound of $e, f$.

Suppose $g \geq e, f$ in $D(A)$. Then $e=e g=g e$ and $f=f g=g f$. So $g(e-f e)=g e-g f e=$ $e-f e$, so $g \geq D(e-f e)$, and so $D(e-f e)=g D(e-f e)$. Hence $g(f+D(e-f e))=$ $g f+g D(e-f e)=f+D(e-f e)$, so $f+D(e-f e) \leq g$. Hence $e \curlyvee f=f+D(e-f e)$ is the least upper bound of $e, f$. 
So strong normality of the D-ring $A$ is necessary and sufficient for $(D(A), \curlywedge, \curlyvee)$ to be a lattice with respect to the standard order on $D(A)$.

For a D-ring with identity $A$, the necessary and sufficient condition for $\curlyvee$, $\curlywedge$ to be lattice operations on $D(A)$ is simply that $A$ is complement-closed, by Corollary 5.1. In that case, we obtain the following.

Corollary 5.10 Suppose $A$ is a complement-closed D-ring with identity. Then $(D(A), \curlyvee, \curlywedge)$ is an orthomodular lattice in which the orthocomplement of $e \in D(A)$ is $e^{\perp}=1-e$.

Proof. For $e \in D(A), e \curlyvee e^{\perp}=(1-e)+D(e-(1-e) e)=1-e+e=1, e \curlywedge e^{\perp}=$ $e-D(e-e(1-e))=e-D(e)=0,\left(e^{\perp}\right)^{\perp}=1-(1-e)=e$, and if $f \in D(A)$ is such that $e \leq f$, then $f^{\perp} \leq e^{\perp}$ is easily checked. So $D(A)$ is orthocomplemented. Finally, if $e, f \in D(A)$ are such that $e \leq f$, then $D(f-e)=f-e$ by Lemma 5.5 , so

$$
\begin{aligned}
e \curlyvee\left(f \curlywedge e^{\perp}\right) & =e \curlyvee(f-D(f-f(1-e))) \\
& =e \curlyvee(f-D(f e)) \\
& =e \curlyvee(f-e) \\
& =e+D((f-e)-e(f-e)) \\
& =e+D(f-e-e f+e) \\
& =e+D(f-e) \\
& =e+(f-e) \\
& =e
\end{aligned}
$$

as required.

Corollary 5.11 Suppose $A$ is a full D-ring with identity. Then $E(A)$ is an orthomodular lattice under $\curlyvee$, ᄉ.

There are special cases of Theorem 5.9 of interest for involuted D-rings, especially in the symmetric case.

Corollary 5.12 Let $A$ be a symmetric involuted D-abundant D-ring. Then $E^{*}(A)$ is a lattice under $\curlyvee$, ᄉ, orthomodular if $A$ has identity.

Proof. If $e f=0$ for $e, f \in D(A)$, then $f e=\left(e^{*} f^{*}\right)^{*}=(e f)^{*}=0^{*}=0$. Also, the D-abundant property implies quasinormality. So $A$ is strongly normal by Lemma 5.4. But $E^{*}(A)=D(A)$ by Lemma 3.2 .

The "with identity" case of this result recovers Lemma 5.3 of [17] (Proposition 1.15 of [3]), applying to Rickart $*$-rings.

The Baer *-semigroups considered in [8] share some of the key features of Rickart *rings. In particular $D(S)$ is an orthomodular lattice; see Theorem 3 there. However, the lattice operations on $D(S)$ as in Theorem 3 of [8] are expressed in terms of a kind of "complementation" operation on $S$ (the so-called focal mapping associated with the focal ideal $K$ of $S$ ), which behaves like $a^{\prime}=1-D(a)$ in a complemented D-ring. Indeed when 
interpreted in this way in a Rickart *-ring $A$, one recovers the lattice operations on $D(A)$. So the result for Baer $*$-semigroups does not seem to have a generalisation to general Dsemigroups, even involuted ones, because of the need to refer to the focal mapping. Even if some variant of the focal mapping is used in such a generalisation, it would not subsume the D-ring without identity case. Theorem 5.9 gives a clean answer to the question of exactly when a D-ring is a lattice under the derived D-ring operations $\curlywedge, \curlyvee$; Corollary 5.10 does the analogous thing for the D-ring with identity case.

\subsection{Subdirect product representations in the D-semiadequate case}

We conclude with consideration of the case of D-semiadequate D-rings; in this case, Theorem 5.9 can be used to obtain subdirect product representations. By Lemma 5.4 and Proposition 5.6, the D-semiadequate D-ring $A$ is strongly normal if and only if it is quasinormal, or equivalently normal satisfying $D(0)=0$.

Theorem 5.13 Let $A$ be a quasinormal D-semiadequate D-ring. Then $D(A)$ is a distributive lattice in which $e \vee f=e \circ f$ and $e \wedge f=e f$.

Proof. Let $A$ be as described. For $e, f \in D(A)$, ef $=f e \leq e$, so $D(e-f e)=D(e-e f)=$

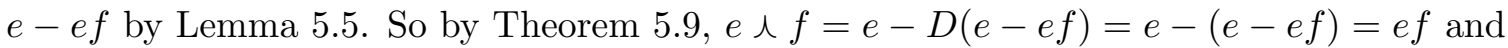
$e \curlyvee f=f+D(e-f e)=f+(e-f e)=e+f-e f=e \circ f$. Distributivity can be shown directly, but in any case follows by first noting that $A$ is a closure ring in the sense of [11] (since $D(A)$ is closed under $\circ$ and $0 \in D(A)$ ) and then applying Proposition 4 there.

Corollary 5.14 Let $A$ be a complement-closed D-semiadequate D-ring. Then $D(A)$ is a Boolean algebra in which $e \vee f=e \circ f$ and $e \wedge f=e f$ and $e^{\prime}=1-e$ is the complement of $e$.

A Boolean ring is a (trivially abelian) complement-closed D-ring if and only if it is a monadic Boolean algebra viewed as a Boolean ring; see [14]. (Monadic algebras are the algebraic models of type S5 modal logic.)

Another example of a quasinormal D-semiadequate D-ring is a direct product of rings with identity $A_{i}, A=\Pi_{i} A_{i}$, in which one defines the operation $D$ by setting $D\left(\left(a_{i}\right)\right)=\left(\delta_{i}\right)$, where $\delta_{i}=0$ if $a_{i}=0$ and $\delta_{i}=1$ otherwise. Call this the coordinate domain operation on the direct product $A$. (That $A$ is a complement-closed D-ring is easily checked.) We return to such examples later.

A $D$-ideal of a D-ring $A$ is an ideal $I$ which satisfies $D(a+i)-D(a) \in I$ for all $i \in I$ and $a \in A$. This is precisely the condition needed to ensure that $A / I$ inherits a well-defined D-ring structure from $A$, if we define $D(a+I)=D(a)+I$, as is easily seen, and then $A / I$ will itself be a D-ring under this operation. Note that in general a D-ideal need not itself be a D-ring, since it may not be closed under the operation $D$; however, if (and only if) $D(0)=0$, then a D-ideal will be a sub-D-ring. Similar comments apply to R-ideals and DR-ideals, defined in the obvious ways.

As noted in the proof of Theorem 5.13, because $D(A)$ is closed under circle composition, the quasinormal D-semiadequate D-ring $A$ is a (left) closure ring in the sense of [11]. It follows that a D-ideal of $A$ is simply an ideal $I$ such that $i \in I$ implies $D(i) \in I$, a socalled "closed ideal" in [11]. As shown there, the D-ideals of such an $A$ are in one-to-one 
correspondence with the normal ideals of the lattice $D(A)$. (They are called normal filters in [11] because the opposite order is used on $D(A)$ compared to the one used here). Note that the strongness property in [11] is implied by the D-abelian property (in fact can be shown equivalent to it in the normal case), and it follows that every ideal of $D(A)$ is normal in the sense used in [11], and that a D-abelian normal D-ring $A$ is simple if and only if $|D(A)|=2$.

Next note that any direct product $A$ of rings with identity equipped with the coordinate domain operation is a quasinormal D-semiadequate (indeed D-abelian) D-ring. In fact it is canonical.

Proposition 5.15 Every non-zero quasinormal D-abelian D-ring $A$ is embeddable in a direct product of rings with identity equipped with its coordinate domain operation.

Proof. Let $I$ be a proper prime ideal of $D(A)$ and let $J=J_{I}$ be the corresponding D-ideal of $A$ (so that $J \cap D(A)=I$ ). In particular, for $e, f \in D(A / J)$, ef $=0$ implies $e=0$ or $f=0$. But for any $e, f \in D(A / J), f(e-e f)=0$, so $f=0$ or $e-e f=0$ (since $A / J$ is quasinormal and $e-e f \in D(A / J)$ as above), that is, $f=0$ or $e \leq f$ for all $e$. Hence $D(A / J)=\{0, i\}$, where $i$ is the largest element of $D(A / J)$ and thus an identity of $A / J$ since $A / J$ is D-abelian. So by Theorem 12 of [11], $A$ is simple, and $e$ is an identity element of $A / J$. So the direct product of the $A / J$ where $J$ ranges over all D-ideals of $A$ of the form $J_{I}$ where $I$ is prime in $D(A)$ is their ring-theoretic direct product equipped with the coordinate domain operation.

By Theorem 5.13, $D(A)$ is a distributive lattice. For every $e \in D(A), e \neq 0$, there is a lattice ideal of $D(A)$ not containing $e$, namely $\{0\}$, and any chain of such ideals has an upper bound with the same property, namely their union, so by Zorn's Lemma there is an ideal of $D(A)$ that is maximal with respect to not containing $e$, say $I$. Moreover any such $I$ is disjoint from the filter generated by $e$ in $D(A)$. Since $D(A)$ is a distributive lattice, $I$ must be a prime ideal. (This was in effect first proved by Stone in [35].) Hence $\bigcap\left\{J_{I} \mid I\right.$ is a prime ideal of $\left.D(A)\right\}=\{0\}$ since any non-zero $e \in D(A)$ fails to be in at least one such $I$. So $A$ embeds into the direct product of the $A / J_{I}$.

If it is assumed that $D(A)$ is a Boolean algebra, this result is a special case of Theorem 13 in [11].

If $A$ is a direct product of domains with identity (rings with identity having no zero divisors), it is easy to see that the coordinate domain operation satisfies the D-abundant condition (and of course is D-semiadequate); hence this applies also to any sub-D-ring of such a direct product. Conversely, we have the following.

Corollary 5.16 Every non-zero D-semiadequate D-abundant D-ring $A$ is embeddable in a direct product of domains with identity equipped with its coordinate domain operation.

Proof. $A$ is abelian by Theorem 4.7, hence D-abelian. Represent $A$ as in the proof of Proposition 5.15. Now if $a b \in J_{I}$ where $I$ is a prime ideal of $D(A)$, then $D(a b) \in J_{I} \cap I=I$. But by the left congruence condition and the D-abelian property, $D(a b)=D(a D(b))=$ $D(D(b) a)=D(D(b) D(a))=D(a) D(b)$. So $D(a) D(b) \in I$, and so $D(a) \in I \subseteq J_{I}$ or $D(b) \in I \subseteq J_{I}$, so $a=D(a) a \in J_{I}$ or $b=D(b) b \in J_{I}$. So $J=J_{I}$ is prime, and $A / J$ has no zero divisors. 


\section{References}

[1] Araujo, J., Kinyon, M. (2012). On a problem of M. Kambites regarding abundant semigroups. Comm. Algebra 40:4439-4447.

[2] Batbedat, A. (1981). $\gamma$-demi-groups, demi-modules, produit demi-directs. In Semigroups, Proceedings, Oberwolfalch, Germany 1978, Lecture Notes in Mathematics 855, Springer-Verlag, pp. 1-18.

[3] Berberian, S.K. (2003). Baer rings and Baer *-rings. The University of Texas at Austin, Registered U.S. Copyright Office March 1988, revised edition.

[4] Bredikhin, D.A. (1977). Abstract characterisation of some classes of binary relation algebras. In Algebra and Number Theory (in Russian), vol. 2, Kabardino-Balkarsk. Gos. Univ., Nalchik, pp. 3-19.

[5] Cockett, J.R.B., Lack, S. (2002). Restriction categories I. Categories of partial maps. Theoret. Comput. Sci. 270:223-259.

[6] Ehresmann, C. (1980-1984). Oeuvres completes et commenties (A.C. Ehresmann, Ed.), Suppl. Cahiers Top Geom. Diff., Amiens.

[7] El-Qallali, A. (1980). Structure Theory for Abundant and Related Semigroups. Ph.D. Thesis, York.

[8] Foulis, D.J. (1960). Baer -semigroups. Proc. Amer. Math. Soc. 11:648-654.

[9] Fountain, J. (1982). Abundant semigroups. Proc. London Math. Soc. 44:103-129.

[10] Fountain, J. (1977). A class of right PP monoids. Quart. J. Math. 28:285-300.

[11] Gardner, B.J., Stokes, T. (1999). Closure rings. Comment. Math. Univ. Carolin. 40:413-427.

[12] Gould, V., Hollings, C. (2009). Partial actions of inverse and weakly left E-ample semigroups. J. Austral. Math. Soc. 86:355-377.

[13] Gould, V., Hollings, C. (2010). Restriction semigroups and inductive constellations. Comm. Algebra 38:261-287.

[14] Halmos, P., Givant, S. (1998). Logic as Algebra. The Mathematical Association of America.

[15] Herstein, I.N. (1976). Rings with involution. Chicago Lectures in Mathematics, University of Chicago Press.

[16] Howie, J.M. (1976). An Introduction to Semigroup Theory. Academic Press, Orlando, FL.

[17] Kaplansky, I. (1951). Projections in Banach algebras. Ann. of Math. 53:235-249. 
[18] Kaplansky, I. (1968). Rings of Operators. Benjamin, New York.

[19] Jackson, M., Stokes, T. (2001). An invitation to C-semigroups. Semigroup Forum 62:279-310.

[20] Jackson, M., Stokes, T. (2003). Agreeable semigroups. J. Algebra 266:393-417.

[21] Jackson, M., Stokes, T. (2011). Modal restriction semigroups: towards an algebra of functions. Int. J. Algebra and Comput. 21:1053-1095.

[22] Jackson, M., Stokes, T. (2013). On representing semigroups with subsemilattices. J. Algebra. 376:228-260.

[23] Lawson, M.V. (1990). Rees matrix semigroups. Proc. Edinburgh Math. Soc. 33:23-37.

[24] Lawson, M.V. (1991). Semigroups and ordered categories I: the reduced case. J. Algebra 141:422-462.

[25] Maeda, S. (1958). On the lattice of projections of a Baer *-ring. J . Sci. Hiroshima Univ. Ser. A 22: 75-88.

[26] Manes, E. (2006). Guarded and Banded Semigroups. Semigroup Forum 72:94-120.

[27] Möller, B., Struth, G. (2006). Algebras of modal operators and partial correctness. Theoret. Comp. Sci. 351:221-239.

[28] Neumann, J. von, (1960). Continuous geometry. Edited by I. Halperin, Princeton Univ. Press, Princeton, N. J.

[29] Preston, G.B. (1954). Representation of inverse semigroups. J. London Math. Soc. 29:411-419.

[30] Schein, B.M. (1970). Relation algebras and function semigroups. Semigroup Forum $1: 1-62$.

[31] Schein, B.M. (1970). A Construction for Idempotent Binary Relations. Proc. Jap. Acad. $46: 246-247$.

[32] Schein, B.M. (1970). Restrictively multiplicative algebras of transformations (in Russian). Izv. Vysš. Učebn. Zaved. 4:91-102.

[33] Schein, B.M. (1979). Lectures on semigroups of transformations. In Amer. Math. Soc. Translat. Ser. 2, 113, pp. 123-181

[34] Schweizer, B., Sklar, A. (1967). Function systems. Math. Annalen 172:1-16.

[35] Stone, M.H. (1936). The theory of representations for Boolean algebra. Trans. Amer. Math. Soc. 40:37-111.

[36] Trokhimenko, V.S. (1973). Menger's function systems (in Russian). Izv. Vyš̌. Učebn. Zaved. Matematika 11:71-78. 
[37] Vagner, V.V. (1952). Generalised groups (in Russian). Proceedings of the USSR Academy of Sciences. 84:1119-1122.

[38] Vagner, V.V. (1953). The theory of generalized heaps and generalized groups (in Russian). Mat. Sbornik N.S. 32:545-632. 